Electron Microscopic and Biochemical Studies of Pyruvate Dehydrogenase Complex of Escherichia coli

By

Humberto Fernández-Morán, M.D., Ph.D.

Biophysics Department, University of Chicago

And

Lester J. Reed, Ph.D.

Masahiko Koike, M.D., Ph.D.

Charles R. Willms, Ph.D.

Clayton Foundation Biochemical Institute and

Department of Chemistry,

University of Texas, Austin

(Submitted for publication to SCIENCE)

June 26,1964 
Electron Microscopic and Biochemical Studies of Pyruvate Dehydrogenase Complex of Escherichia coli 29416

Abstract: Examination of the Escherichia coll pyruvate dehydrogenase complex and its component enzymes in the electron microscope indicates that the complex has a polyhedral structure with a diameter of about 300 to $350 \AA$ and a height of 200 to $250 \AA$. The lipoic reductase-transacetylase aggregate, consisting of about 64 subunits, occupies the central portion of the polyhedron and has the appearance of a tetrad of 130 to $150 \AA$. Surrounding this tetrad are about 16 molecules of pyruvate decarboxylase and about 8 molecules of dihydrolipoic dehydrogenase arranged into two rings laid one above the other. The sequence of these latter molecules cannot yet be specified. However, many of these molecules are similar in appearance and dimensions (70 to $90 \AA$ ) to those observed in electron micrographs of the isolated pyruvate decarboxylase component of the complex. The reconstituted complex closely resembles the native complex in appearance.

Autho 


\section{Electron Microscopic and Biochemical studies of Pyruvate Dehydrogenase Complex of Escherichia coli}

The Escherichia coli pyruvate dehydrogenase complex (PDC) is a highly organized multienzyme system, with a molecular weight of approximately 4.8 million, that catalyzes a multistep oxidative decarboxylation of pyruvate $(1,2)$, consisting of a decarboxylation, reductive acetylation (acetyl generation), acetyl transfer, and electron transfer. Because of the wealth of available biochemical data on this multienxyme complex, a unique opportunity is provided to correlate functional properties, as revealed by biochemical analysis, with ultrastructure, as revealed by electron microscopy. The picture of the structural organization of PDC which emerged from biochemical studies is that of an organized mosaic of enzymes in which each of the component enzymes is uniquely located to permit efficient implementation of the consecutive reaction sequence. This picture has been confirmed and extended by correlative electron microscope studies which are reported in the present communication.

PDC is composed of three enzymes: (i) pyruvate decarboxylase, (ii) lipoic reductase-transacetylase (IRT), and (iii) a flavoprotein, dihydrolipoic dehydrogenase. LRT is an aggregate of a subunit with a molecular weight of about $27,000(2,3)$. The composition of PDC is shown in Table 1.

Table I

Composition of Pyruvate Dehydrogenase Complex

Component

Pyruvate decarboxylase

Dihydrolipoic dehydrogenase

Lipoic reductase-transacetlyase subunit

\begin{tabular}{cc}
$\begin{array}{c}\text { Molecular } \\
\text { Weight }\end{array}$ & $\begin{array}{c}\text { Number of Molecules Per } \\
\text { Molecule of Complex }\end{array}$ \\
\cline { 2 - 2 } 183,000 & 16 \\
112,000 & 8 \\
27,000 & 64
\end{tabular}

The methods employed in resolving PDC into its component enzymes and in reconstituting PDC from the isolatcd enzymes 


\section{Electron Microscopic and Blochemical Studies of}

Py ruvate Dehydrogenase Complex of Escherichia coli

have been described (2). Briefly, selective dissociation of the flavoprotein from the pyruvate dehydrogenase complex $\left(\mathrm{s}_{20, \mathrm{w}}\right.$ about $60 \mathrm{~s}$ ) is accomplished by fractionation in the presence of $4 \mathrm{M}$ urea on calcium phosphate gel suspended on cellulose. This procedure yields free flavoprotein $\left(\mathrm{s}_{20}, \mathrm{w}=6.3 \mathrm{~s}\right.$ ) and a complex of decarboxylase and LRT $\left(\mathrm{s}_{20, \mathrm{w}}\right.$ about $\left.50 \mathrm{~s}\right)$. The latter complex is separated into the decarboxylase $\left(s_{20, w}=9.2 s\right)$ and LRT ( $s_{20, W}$ about $27 \mathrm{~s}$ ) by fractionation on gel-cellulose at $\mathrm{pH}$ 9.5. $\mathrm{PDC}$ is reconstituted by mixing the isolated decarboxylase, LRT aggregate, and flavoprotein in $0.05 \mathrm{M}$ potasium phosphate buffer $(\mathrm{pH} 7.0)$ in a ratio of $3: 2: 1$ by weight, respectively. Examination of this mixture in a spinco model $E$ ultracentrifuge reveals the virtual absence of peaks corresponding to the individual components. Instead, a major, faster moving peak $\left(s_{20, w}=52.4 \mathrm{~s}\right)$ is observed with which the yellow color of the flavoprotein is associated. This major component is isolated as a yellow pellet by centrifugation for $2-1 / 2$ hours at $173,000 \mathrm{xg}$. The composition and enzymatic activities of the yellow pellet are very similar to those of native $\mathrm{PDC}(2)$.

Electron microscopy: Negative staining-embedding of specimens was achieved by the microdroplet cross-spraying technique (4). Employing a special multiple-spraying device provided with suitably arranged separate capillaries for specimens and reagents, it is possible to obtain controlled brief interaction of microdroplets of the specimen with microdroplets of 1 to 2 percent potassium phosphotungstate at $\mathrm{pH} 7.2$ to 7.4. The cross-sprayed microdroplets (ca. 0.1 to $10 \mu$ diameter) collide and interact very rapidly shortly before impinging on the specimen grid. As a result of the small size and relatively high speed of the cross-sprayed microdroplets, there is a favorable concurrence of rapid specimen cooling and limited 


\section{Electron Microscopic and Biochemical. Studies of Pyruvate Dehydrogenase Complex of Escherichia coli}

drying to attain a unique degree of preservation of labile structures $(4,5)$. Positive staining with 1 or 2 percent uranyl acetate was also accomplished by this technique. All reagents were ultrafiltered using 100 \& Millipore filters. Operations were carried out at $0-5^{\circ}$. Specimens were examined in the native state shortly after preparation, or after freezing and thawing under controlied conditions without undergoing significant loss in activity. Final concentration of the specimens was generally of $0.5 \mathrm{mg} / \mathrm{ml}$. protein in $0.02-0.05 \mathrm{M}$ potassium phosphate buffer. other buffers, e.g. ammonium acetate were used, with similar results. Extensive control experiments were carried out with standard preparation techniques. The specimens were mounted on very thin Formvar films, or supported on special fenestrated specimen substrates (4). A Siemens Elmiskop I was used with multiple objective aperatures and pointed filament of singlecrystal tungsten $(4,6)$ providing microbeam illumination of high coherence. The micrographs were recorded at electron optical magnif $\perp$ cations of $x 20,000$ to $X 80,000$ on Ilford high resolution plates. Ferritin molecules of uniform size ( 118 to $120 \AA$ diameter) prepared by density gradient ultracentrifugation were included as an internal calibration reference. Irradiation damage and specimen contamination were substantially reduced with improved specimen cooling devices and low-intensity electron optics $(4,5)$.

A typical field of PDC particles negatively stained with phosphotungstate is shown in Fig. 1. The PDC particles have the appearance of polyhedrons of diameter about 300 to $400 \%$. There are four well-defined subunits in the central portion of each polyhedron, arranged as a tetrad with a side of 130 to $150 \AA$. Surrounding this central tetrad is an orderly array of subunits, 60 to $90 \AA$ in diameter. The appearance of some of the projections 


\section{Electron Microscopic and Blochemical Studies of}

\section{Pyruvate Dehydrogenase Complex of Escherichia coli}

of negatively and positively stained preparations suggests a double layer of these peripheral subunits. The relatively large variation in the diameter of the PDC particles, i.e. 300 to $400 \AA$, as seen in negative contrast appears to be due mainly to swelling of the particles caused by phosphotungstate. Ultracentrifuge studies indicate that phosphotungstate is adsorbed by the PDC particles, and that some separation of subunits occurs. The electron micrographs indicate a tendency for the peripheral subunits of PDC to dissociate from the complex in the presence of phosphotungstate. Heavy staining of the PDC particles by uranyl acetate, indicated by the electron micrographs, also would result in larger than actual dimensions. In view of these complications, the minimal dimensions observed, 300 X $200 \AA$, are believed to represent a reasonable estimate of the actual dimensions of the PDC particle.

The pyruvate dehydrogenase complex reconstituted from the isolated decarboxylase, LRT aggregate and flavoprotein is shown in negative contrast in Fig. $2 \mathrm{~B}$. The central tetrad of the complex stands out clearly, and the peripheral subunits are also well-defined. Comparison with Figs. 1,2A reveals the virtual identity of appearance of the reconstituted and native complexes. This supports the biochemical data indicating that the sedimentation characteristics, composition and enzymatic activities of the reconstituted and native PDC are very similar, and points up the uniqueness of the structural organization of PDC.

Figs. 2C,D show different projections of the pyruvate dehydrogenase complex positively stained with 2 percent uranyl acetate. The appearance of the central LRT tetrad is not as distinct in these micrographs as in the negatively stained preparations. However, the individual peripheral subunits are more clearly outlined. Many of the latter subunits have the appearance of oval rings with a diameter of 70 to $90 \AA$. Fig. 20 
Electron Microscopic and Biochemical Studies of Pyruvate Dehydrogenase Complex of Escherichia coli

represents a side view of the pyruvate dehydrogenase complex. A double layer of peripheral subunits is clearly demonstrated in this micrograph. The width of the complex in this projection is 200 to $250 \AA$.

The appearance of the isolated pyruvate decarboxylase positively stained with uranyl acetate is shown in Fig. $2 E$. This projection of the molecule looks like an oval ring of diameter 70 to $90 \AA$ with a dark spot of diameter about $15 \AA$ in the center. The former dimension is in reasonable agreement with a spherical diameter of $75 \AA$ calculated from the molecular weight of pyruvate decarboxylase. The appearance and dimensions of pyruvate decarboxylase as seen in positive contrast closely resemble those of some of the repeating subunits along the periphery of PDC (Figs. 2C,D).

Electron micrographs of the isolated LRT negatively stained with phosphotungstate show arrays of tetrads (Fig $2 F$ ). These tetrads have the same average dimension (130 to $150 \AA$ ) as and closely resemble the central tetrad of the native PDC (Figs. 1,2). The dense core of the tetrads may be partly attributed to penetration of the phosphotungstate.

On the basis of the biochemical and electron microscopic data, a tentative model of the pyruvate dehydrogenase complex has been constructed (Fig. 3). The component enzymes of the complex are represented as spheres, the diameters of which were calculated from the molecular weights of the enzymes and an assumed partial specific volume of $0.73 \mathrm{ml}$ per gm. These diameters are 75,40 and $64 \AA$ for the decarboxylase, LRT subunit and flavoprotein, respectively. The model consists of 16 molecules of pyruvate decarboxylase (large spheres) and 8 molecules of flavoprotein (medium-size spheres) arranged 
Electron Microscopic and Biochemical Studies of Pyruvate Dehydrogenase Complex of Escherichia coli

into two rings laid one above the other. These two rings surround the lipoic reductase-transacetylase aggregate which comprises 64 subunits (small spheres) arranged into four stacks. We cannot as yet specify the sequence of pyruvate decarboxylase and dihydrolipoic dehydrogenase molecules in the two rings.

The volume of PDC calculated from electron microscope measurements (minimal dimensions $300 \times 200 \AA$ ), assuming that the shape of the particle may be approximated as either an oblate ellipsoid or a right circular cylinder, is about $9.5 \times 10^{-18} \mathrm{~cm}^{3}$. The anhydrous volume of PDC calculated from hydrodynamic data (1) is about $5.8 \times 10^{-18} \mathrm{~cm}^{3}$. The approximately 64 percent difference between the two volumes is attributed to the open structure of PDC, indicated by the electron micrographs and represented schematically by the model. This interpretation adequately explains the high frictional ratio $\left(f / f_{0}\right)$ of $P D C$, which has been calculated to be 1.6 .

Correlated studies of this multienzyme complex may prove to be of particular operational significance in elucidating the functional organization of other specific macromolecular assemblies in the living cell. 
Electron Microscopic and Biochemical studies of Pyruvate Dehydrogenase Complex of Escherichia coli

Humberto Fernández-Morán*

Department of Biophysics

University of Chicago, Chicago

Lester J. Reed**

Masahiko Koike***

Charles R. Willms

Clayton Foundation

Biochemical Institute and Department of Chemistry

University of Texas, Austin

* Supported by U.S. Atomic Energy Commission contract AT (30-1)2278 , by grants $\mathrm{B}-2460, \mathrm{C}-3174$, and $\mathrm{NB}-04267$ from the National Institutes of Health, and by NASA Grant NsG 441-63 from the National Aeronautics and Space Administration.

* Supported in part by grant GM-06590 from the National Institutes of Health.

*** Present address of Masahiko Koike: Atomic Disease Institute, Nagasaki University School of Medicine, Nagasaki, Japan.

Lester J. Reed acknowledges valuable discussions with Dr. D. M. Ziegler and Dr. D. J. Cox of the Department of Chemistry, University of Texas, and Dr.W. R. Carroll, National Institute of Arthritis and Metabolic Diseases.

\section{REFERENCES AND NOTES}

I. (a) M. Koike, L. J. Reed, W. R. Carroll, J. Biol. Chem. 235, 1924 (1960); (b) M. Koike and L. J. Ree $\frac{\text { ibi }}{d}$, p. 1931.

2. M. Koike, I. J. Reed, W. R. Carroll, ㅌid. 238, 30 (1963).

3. C. R. Willms and L. J. Reed, Federation Proc. 23, 264 (1964).

4. H. Fernández-Morán, Circulation 26, 1039 (1962).

5. H. Fernández-Morán, J. Royal Microscop. Soc. 83, 5 (1964).

6. H. Fernández-Morán, J. Appl. Phys. 31, 1840 (1960). 


Electron Microscopic and Biochemical Studies of
Pyruvate Dehydrogenase Complex of Escherichia coli

\section{Texts of Illustrations}

Fig. I: Pyruvate dehydrogenase complex of Escherichia coli negatively stained with 1 percent phosphotungstate ( $\mathrm{pH} 7.4$ ) using microdroplet cross-spraying technique. (X 520,000).

Fig. 2: Electron micrographs of E. ooli pyruvate dehydrogenase complex (PDC): (A) Native PDC; (B) Reconstituted PDC with Ferritin negatively stained with phosphotungstate; (C)(D) Native PDC positively stained with uranyl acetate; (E) Isolated pyruvate decarboxylase stained with uranyl acetate; (F) Isolated LRT aggregate negatively stained. (X 700,000).

Fig. 3: Tentative model of E. coli pyruvate dehydrogenase complex. 


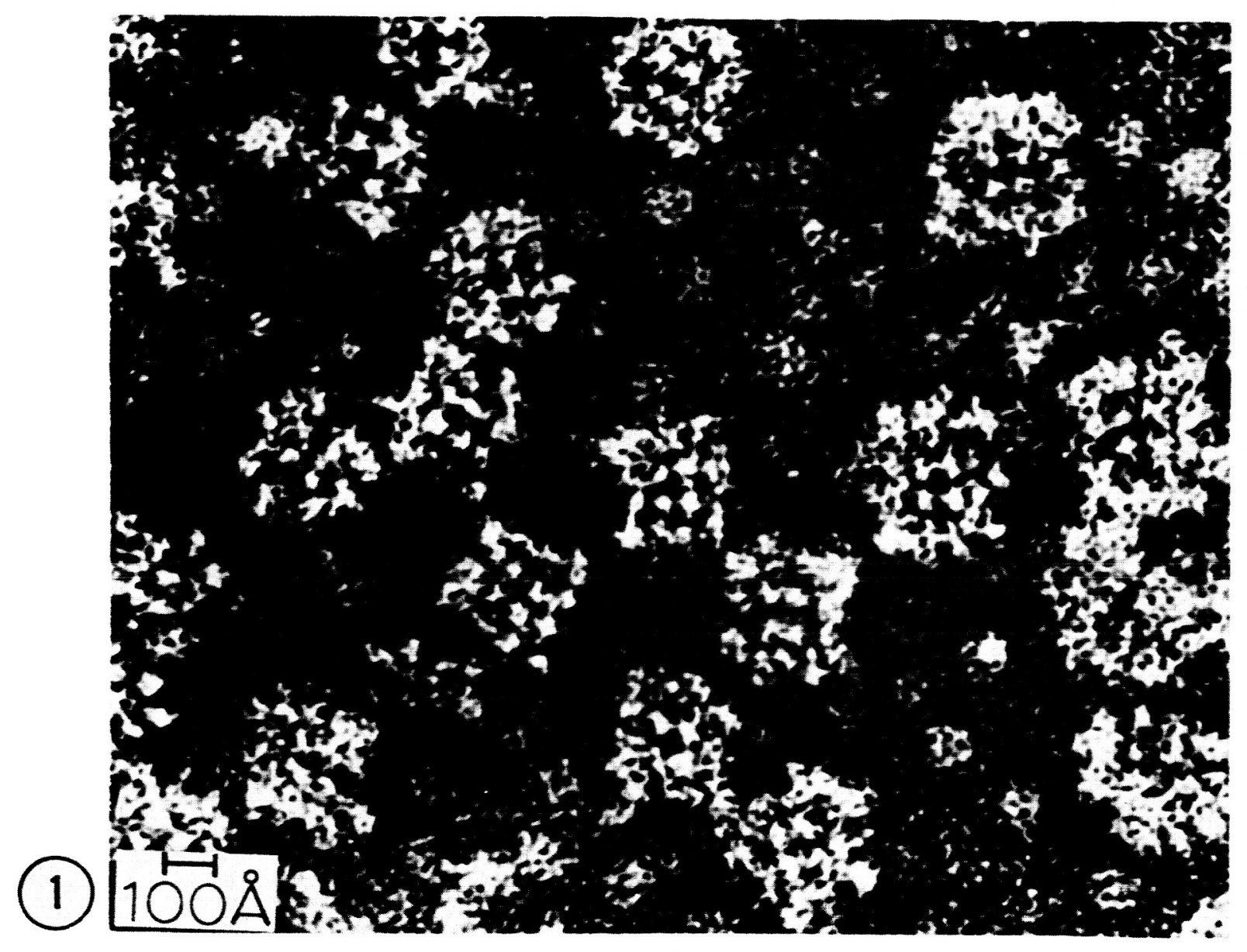



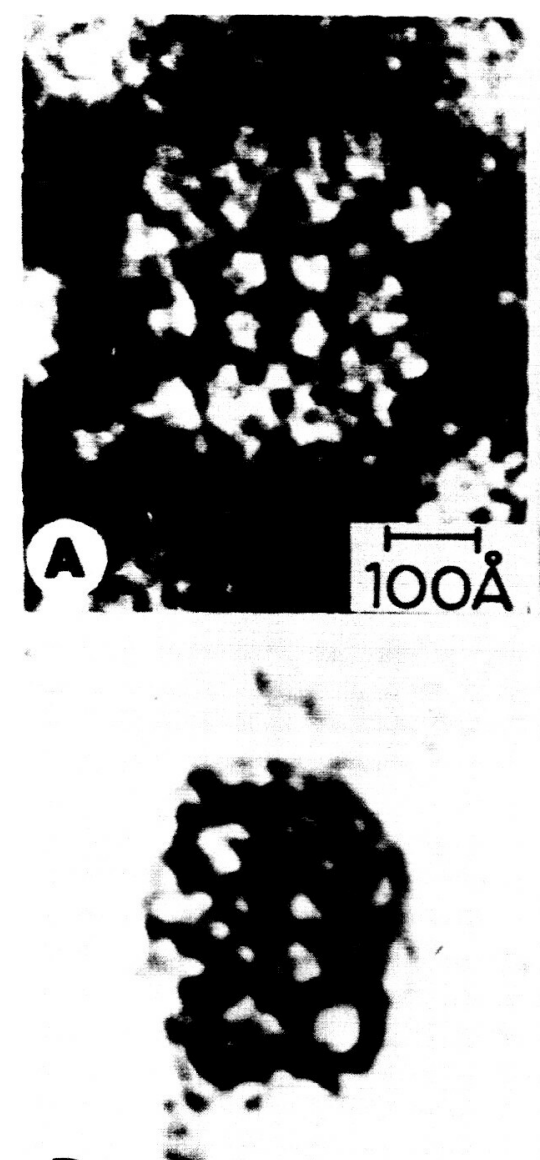

(2)
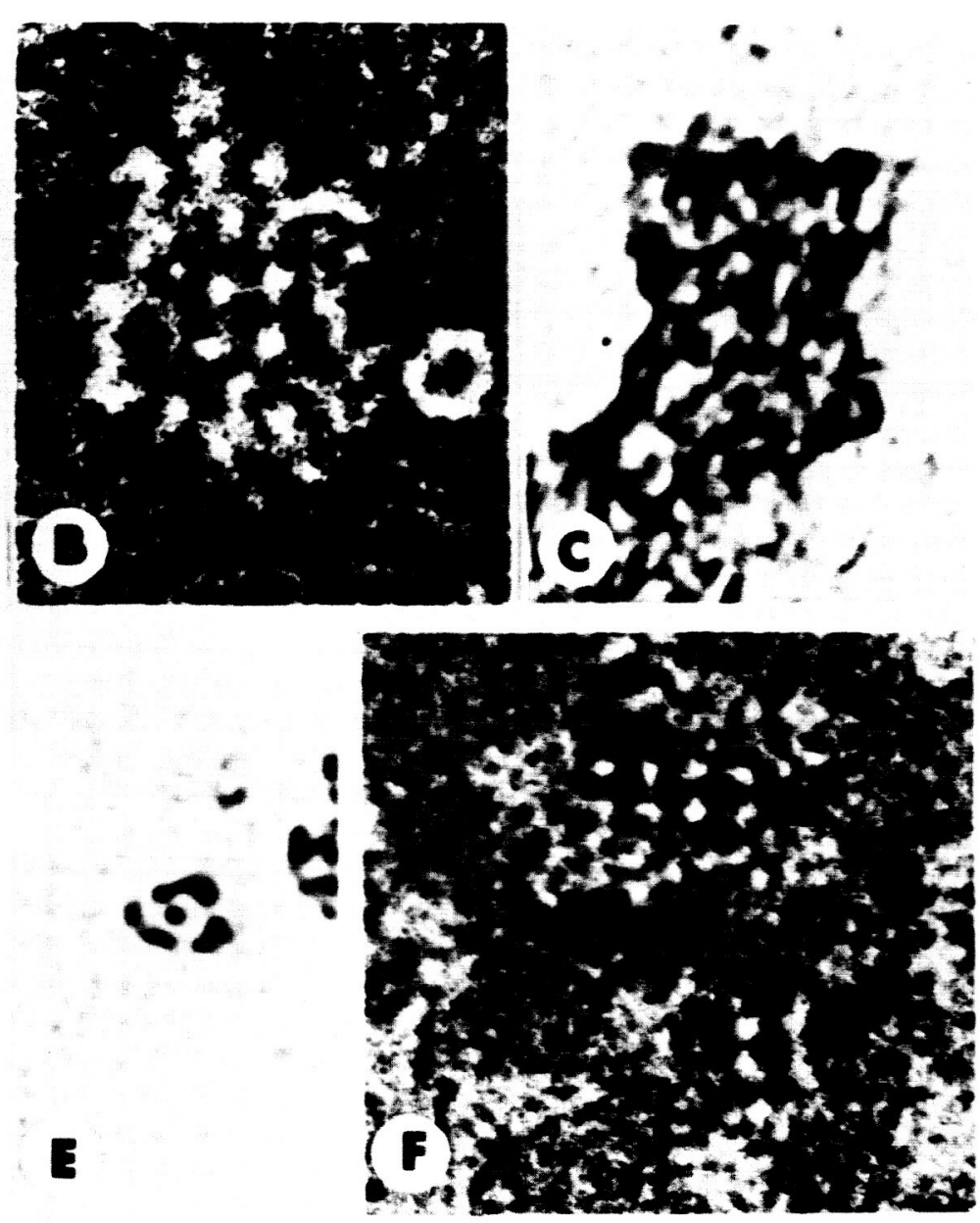

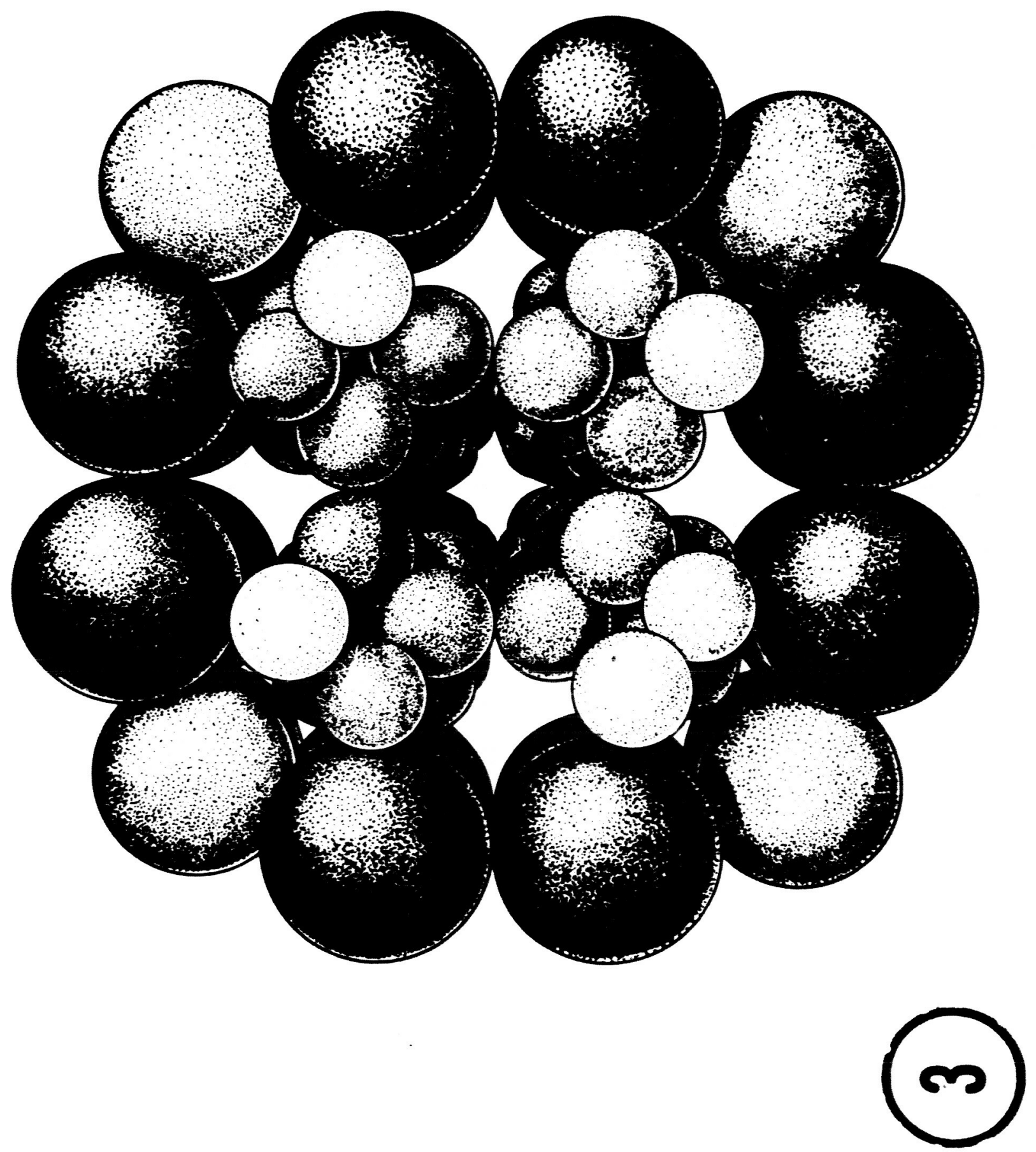